\title{
Le LHC, grand collisionneur de hadrons(l)
}

Bruno Mansoulié (bruno.mansoulie@cea.fr)

Atlas Team Leader, IRFU, Service de physique des particules, CEA/Saclay, 91191 Gif-sur-Yvette

Après plus de 15 ans d'efforts, le 10 septembre 2008 , le CERN a mis en service à Genève, le LHC, le plus puissant accélérateur de particules jamais construit. Nous sommes convaincus que cet instrument, quand il fonctionnera à plein régime, apportera une contribution décisive à notre connaissance des structures élémentaires de la matière.

En effet, on espère au moins compléter le Modèle Standard de la physique des particules, en découvrant le boson de Higgs, toujours inobservé. Mais nombreux sont ceux qui pensent que

l'expérimentation au LHC pourrait bien ouvrir une fenêtre sur un domaine totalement nouveau, et commencer de répondre aux grandes questions de la physique d'aujourd'hui : d'où vient la masse des particules ? Quelle est la nature de la matière noire ?

(1) Hadron : particule composite sensible à l'interaction nucléaire forte. Les hadrons sont composés de quarks, d'antiquarks et de gluons. Le proton, le neutron et leurs antiparticules, ainsi que les mésons, sont des hadrons.

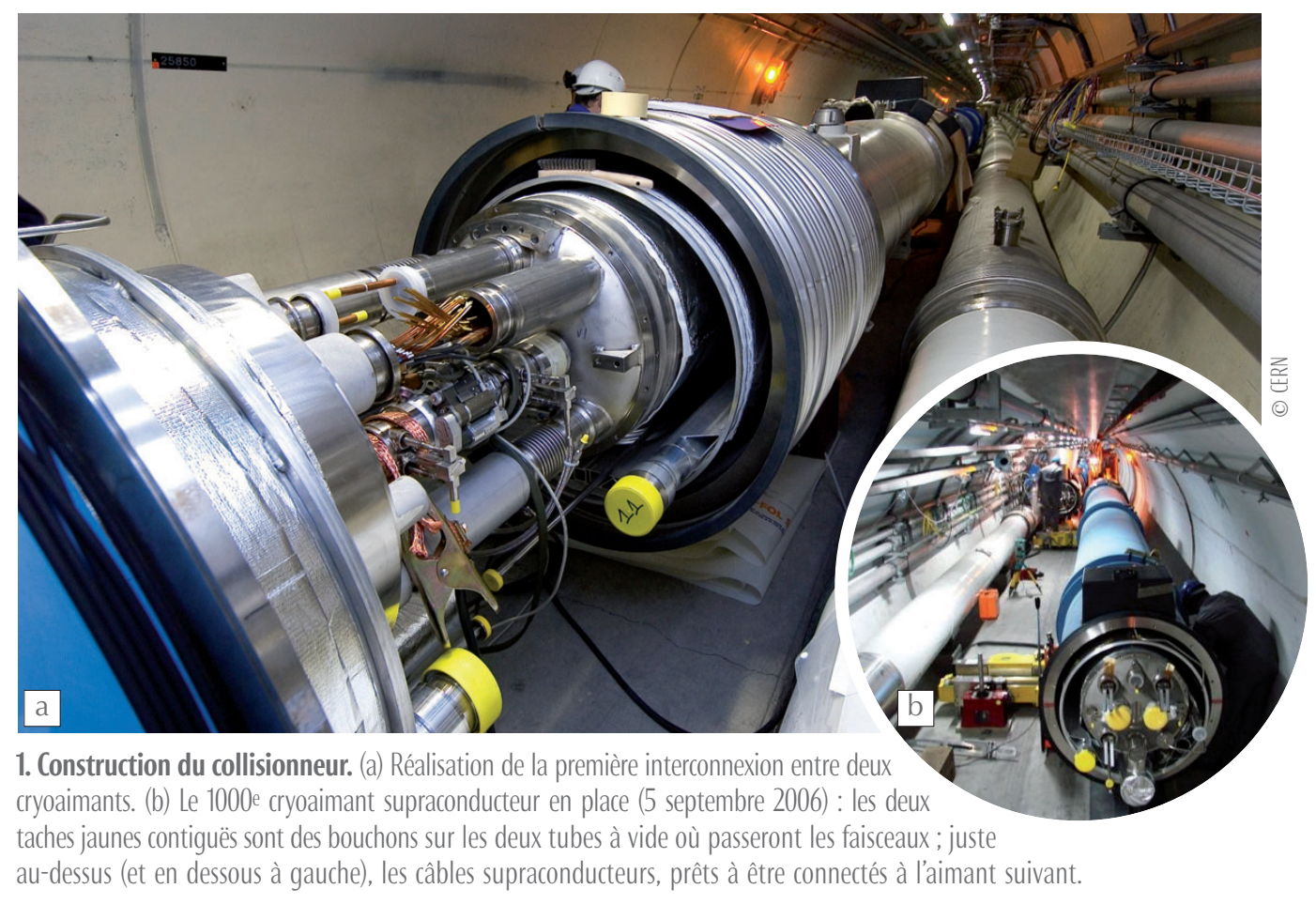

L'origine du projet LHC (Large Hadron Collider) remonte au début des années 1980 [1]. À ce moment, le CERN envisage de construire un grand collisionneur électron-positron, le LEP. La taille de l'anneau doit être aussi grande que possible, dans les limites du budget. Dès le début, certains pensent qu'après le LEP, le tunnel pourrait accueillir un anneau de protons. La circonférence du tunnel est fixée à 27 km. En 1984, les USA font le projet d'un collisionneur de protons de $80 \mathrm{~km}$ de circonférence. Ce chiffre est là pour s'assurer de dominer la future possibilité du CERN, mais aussi parce qu'avec la technologie d'aimants supraconducteurs de l'époque (atteignant un champ de 4T), c'est la dimension requise pour explorer l'échelle d'énergie "magique " de $1 \mathrm{TeV}(1000 \mathrm{GeV})$ dans les collisions.

Le CERN construit donc le LEP et, en parallèle, lance un programme de R\&D intense dans deux directions : tout d'abord, augmenter le champ des aimants de l'accélérateur pour augmenter l'énergie des faisceaux, à tunnel fixé. La valeur atteinte, qui sera utilisée par le LHC, est de 8,4T, plus de deux fois la valeur des machines précédentes. Ensuite, augmenter l'intensité des faisceaux, et surtout développer des détecteurs capables de mesures très diverses et de précision dans un environnement difficile : collisions très rapprochées (25 ns) et nombreuses, rayonnements importants.

En 1993, les USA abandonnent leur projet puis rejoignent le LHC, suivis par d'autres pays non-membres du CERN (Japon, Canada, Inde...). (Voir l'encadré 1, p. 7, qui donne quelques dates et données clés sur le LHC.)

\section{L'accélérateur}

Le LHC est donc un " collisionneur " de protons : il comprend deux tubes à vide (fig. 1) dans lesquels circulent des paquets de protons, en sens inverse. Les anneaux se croisent en huit points ; en quatre de ces points sont installées les expériences qui observent les particules issues des collisions de protons. (Voir, à titre d'illustration, le schéma de la chaîne d'accélération du Fermilab aux USA, p. 11.)

Les paquets de protons (environ $310^{11}$ protons, $4 \mathrm{~cm}$ de long) sont guidés par des aimants supraconducteurs : 1232 dipôles pour la courbure, 392 quadripôles pour la focalisation, et des milliers de petits aimants correcteurs (sextupôles, 


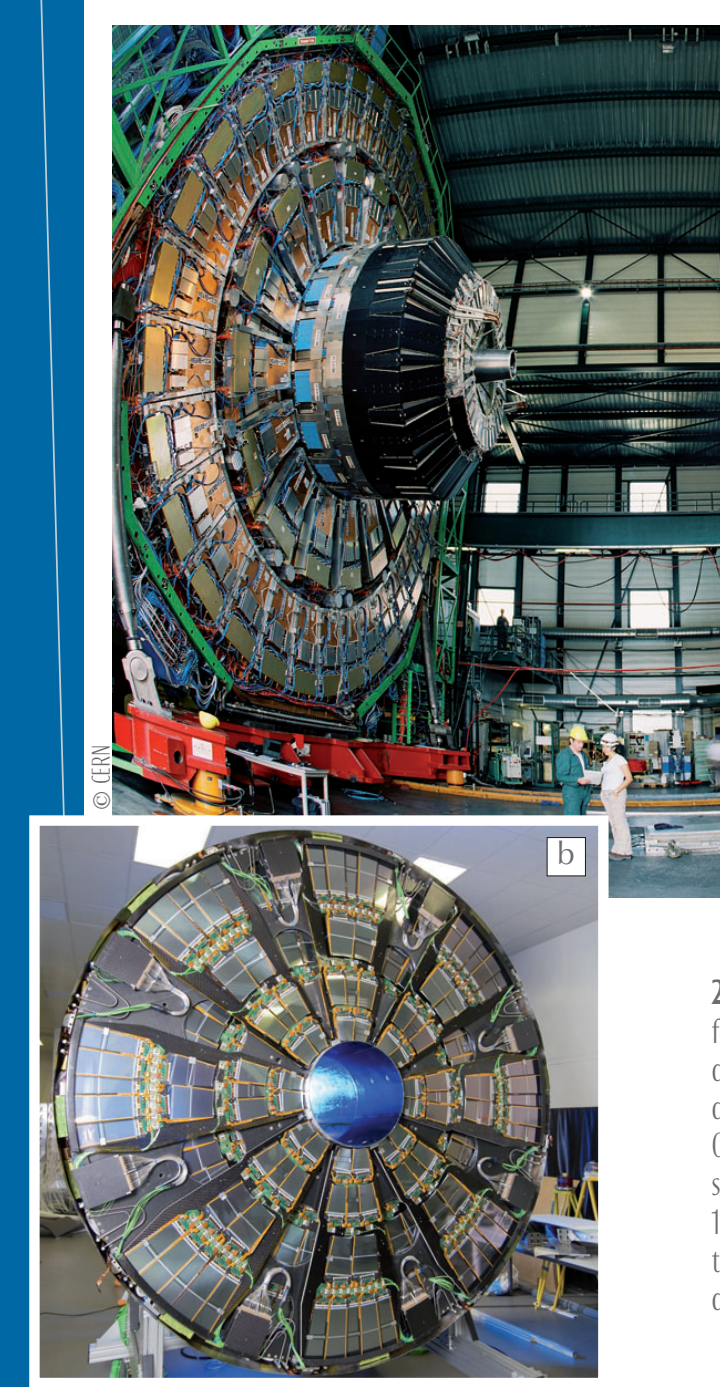

$>>>$

octupôles...). Les dipôles de $15 \mathrm{~m}$ de longueur (cylindres bleus sur l'image de couverture) comprennent deux ouvertures pour les deux faisceaux, avec des champs magnétiques opposés qui se referment l'un l'autre. Les aimants sont refroidis à $1,9 \mathrm{~K}$ par de l'hélium superfluide, permettant d'utiliser plus de courant (donc de champ) qu'à la température de l'hélium liquide (4 K).

À pleine intensité, 2800 paquets de protons circuleront dans chaque anneau (environ un paquet tous les 8 mètres). Les croisements de paquets se produiront donc aux points d'intersection toutes les $25 \mathrm{~ns}$ (40 MHz). Aux points d'intersection utilisés par les expériences, les faisceaux sont focalisés pour obtenir la taille transverse la plus petite possible, environ 15 microns, afin de provoquer le maximum de collisions.

L'énergie stockée dans les faisceaux est très importante, $360 \mathrm{MJ}$ par faisceau, soit environ $60 \mathrm{~kg}$ de TNT. Un des défis de la machine est donc le contrôle des faisceaux, la détection de tout défaut de trajectoire, et l'extraction rapide d'un faisceau sur une zone d'abandon sûre.
2. Le détecteur de l'expérience CMS. (a) Avant fermeture pour test par mesure du passage des muons du rayonnement cosmique. (b) Une des parties " avant " du détecteur interne de traces. Diamètre : environ $2 \mathrm{~m}$. On voit les " pétales ", constitués de plaquettes de silicium. Sur chaque plaquette sont gravées environ 1000 pistes de détection lues individuellement. La surface totale de silicium du détecteur interne de CMS est d'environ $200 \mathrm{~m}^{2}$ pour 75 millions de pistes et pixels.

\section{Les expériences}

Une « expérience » est en fait un observatoire de collisions. L'accélérateur fournit des collisions ("événements "), que l'expérience trie, enregistre, reconstruit, analyse. La statistique joue ici un grand rôle : dans un croisement de paquets, les collisions proton-proton se produisent au hasard, et leur nombre fluctue autour du nombre moyen. Ensuite, une collision de deux protons peut être plus ou moins "dure ", en mécanique classique on dirait plus ou moins «frontale ». Enfin, au niveau quantique, le même état initial (la même collision) peut produire des états finals différents, suivant une loi de probabilité.

On utilise des protons parce qu'il est possible de les accélérer à des énergies très élevées, bien plus que des électrons. Mais il $\mathrm{y}$ a un prix à payer : les protons ne sont pas des particules élémentaires, mais sont constitués de plusieurs quarks et de gluons liés les uns aux autres, qui se partagent aléatoirement l'impulsion du proton. Les collisions les plus dures seront donc celles entre un composant (quark, gluon) d'un proton et un composant de l'autre. Ceci a plusieurs conséquences importantes : tout d'abord, l'énergie mise en jeu dans la collision "élémentaire " varie d'une collision à l'autre. Ensuite, les composants " spectateurs " des protons ne restent pas totalement inactifs ! Ils sont à l'origine d'un grand nombre de particules " molles » qui s'ajoutent à celles produites par la collision "dure ": c'est l'événement sous-jacent.

Parmi les quatre expériences, Atlas et CMS sont des détecteurs polyvalents, couvrant tout l'angle solide autour du point de collision. Ils visent à explorer la physique des collisions les plus dures (et donc les plus rares), à des énergies jamais atteintes auparavant. Nous reviendrons sur ce programme de physique.

L'expérience LHCb étudie aussi les collisions proton-proton, mais se spécialise dans l'étude des états finals comportant des quarks $b$ et les mesures de précision.

Il sera aussi possible d'accélérer des ions dans le LHC (au cours de périodes dédiées), et l'expérience Alice est spécialisée dans l'étude des collisions ion-ion (jusqu'à plomb-plomb), et la mise en évidence d'un nouvel état de la matière nucléaire : le plasma de quarks et de gluons.

Atlas et CMS sont typiques des expériences polyvalentes sur les collisionneurs, mais à des tailles beaucoup plus grandes que celles des générations précédentes (au LEP par exemple). Atlas mesure $26 \mathrm{~m}$ de diamètre et $45 \mathrm{~m}$ de long, et pèse $\sim 7000 \mathrm{t}$; CMS (fig. 2) est plus compacte, mais pèse 13000 t. Toutes deux présentent une structure en oignon autour du point de collision, chaque couche étant constituée d'une partie centrale cylindrique et de deux «bouchons ». En partant du point de collision et en allant vers l'extérieur, on trouve (voir encadré 2, p. 8)

- Le détecteur central, qui détecte en plusieurs points le passage des particules chargées, en les perturbant le moins possible. Il comprend un très grand nombre de détecteurs indépendants très précis (plusieurs millions), pour pouvoir séparer et mesurer toutes les particules issues des collisions (fig. 2). - Le calorimètre électromagnétique, qui présente un milieu très dense dans lequel interagissent les particules, en particulier les électrons (et positrons) et photons. Les particules secondaires ré-interagissent à leur tour, formant des gerbes assez localisées dans le détecteur (quelques dizaines de $\mathrm{cm}$ de long sur quelques $\mathrm{cm}$ de diamètre). La partie chargée de ces gerbes est détectée, le signal total étant proportionnel à l'énergie de la particule initiale. 
- Le calorimètre hadronique, dense lui aussi, qui remplit la même fonction pour les autres types de particules (pions, kaons, protons, etc.)

- Le détecteur à muons : après les calorimètres, les seules particules qui ont traversé sont les muons (le muon est un " frère " de l'électron, de masse plus élevée). Leur passage est détecté par des chambres d'ionisation à fils.

Le détecteur central et le détecteur à muons sont plongés dans des champs magnétiques intenses. La mesure de la courbure de la trajectoire des particules chargées permet la détermination de leur impulsion.

Les deux expériences Atlas et CMS different cependant considérablement. Tout d'abord, les configurations de leurs champs magnétiques sont totalement différentes. Ensuite, les technologies choisies pour chacun des détecteurs cités ci-dessus sont différentes. Ces choix correspondent à des approches différentes des risques et des possibilités d'erreur de mesures. Il était indispensable d'avoir deux expériences concurrentes et totalement indépendantes pour garantir les résultats ; il est intéressant que les choix techniques soient aussi complémentaires.

Chacune des quatre expériences du LHC a été conçue et réalisée par une collaboration de 100 à 200 instituts du monde entier, rassemblant de 1000 à 2000 physiciens et leurs supports techniques. Les modes de communication, décision, management, de telles collaborations, essentiellement autogérées, sont particulièrement intéressants, mais ce serait le sujet d'un autre article...

\section{Environnement expérimental, analyse, simulation}

Les collisions " ordinaires " entre deux protons, régies en premier lieu par l'interaction nucléaire forte, ont une probabilité élevée, et produisent de nombreuses particules. En fonctionnement nominal, chaque croisement de paquets de protons sera l'occasion d'une vingtaine de collisions en moyenne. Chaque collision produira en moyenne une centaine de particules, distribuées dans tout l'angle solide. Au total, chaque croisement, toutes les $25 \mathrm{~ns}$, engendre plus d'un millier de particules. Les détecteurs doivent donc être très rapides, pour ne pas confondre les événements de croisements consécutifs, et finement divisés, pour mesurer chacune des particules. Ce flux de particules impose aussi l'emploi de détecteurs et d'électronique résistant aux radiations.
Les événements « banals », issus de collisions " molles ", sont extrêmement nombreux (un milliard par seconde). En revanche, les événements "intéressants " sont produits dans la collision de très haute énergie entre deux constituants de l'un et l'autre proton, lorsque chacun de ces constituants emporte une grande fraction de l'impulsion de son proton parent. La probabilité qu'un constituant emporte une impulsion élevée est très faible, et ces collisions " dures " sont donc très rares.

Le potentiel de découverte de nouvelles particules, ou d'un nouvel effet, dépend donc directement de la capacité d'une expérience à isoler de très rares événements dans un grand "bruit de fond » d'événements banals provenant d'une physique déjà connue.

Dans les cas les plus favorables, il est possible d'annoncer une découverte en ayant observé une dizaine d'événements bien identifiés, si on a prouvé que le bruit de fond (plusieurs milliards d'événements au départ), peut être réduit à moins d'un ou deux événements.

Pour concevoir le détecteur et pour imaginer une " analyse ", on peut partir d'un modèle théorique, explorer les événements qu'il prédit et leurs caractéristiques, et faire en sorte qu'après mesures et analyse, les données montrent un excès d'événements

\section{Le LHC en bref}

Quelques dates :

- 1984 : première réunion à Lausanne

- 1991 : résolution de principe sur le LHC

- 1994 : accord des pays européens (membres du CERN) pour le LHC

- 1995 : approbation des expériences Atlas et CMS

- 1997 : USA, Japon et Inde rejoignent le LHC

- 1998 : début du génie civil des cavernes des expériences

.. Conception et construction de l'accélérateur et des expériences

- 2008 : mise en service

Quelques données :

- Anneau de collision proton-proton

- Circonférence : 27 km

- Énergie nominale par faisceau : $7 \mathrm{TeV}$

- Dans chaque sens : 2808 paquets de $10^{11}$ protons

- 4 points d'interactions avec 4 expériences : Atlas, CMS, LHCb, Alice

- Fréquence des collisions : $40 \mathrm{MHz}$

- Taux d'événements enregistrés par expérience : $200 \mathrm{~Hz}$

- Taille d'un événement enregistré : environ 1,5 MByte

- Information enregistrée, à analyser, par an par expérience : $10^{7}$ GByte

La participation française

- Pour I'accélérateur : conception et industrialisation des quadripôles (CEA-IRFU, CNRS-IPNO) ; cryogénie He superfluide (CEA-SBT).

- Pour les expériences :

Les équipes de physiciens, ingénieurs et techniciens du CEA-IRFU et du CNRS-IN2P3

(11 laboratoires) ont pris une part très importante à la conception, construction et mise en œuvre des détecteurs. Aujourd'hui, environ 250 physiciens de ces laboratoires préparent I'analyse des données. 
Ce mécanisme permet l'unification des interactions électromagnétique et faible, et, au passage, est à l'origine des masses de toutes les particules élémentaires, constituants ou messagers [2].

Le boson de Higgs est la seule particule du Modèle Standard non encore observée. Sa masse $m_{H}$ est inconnue : le LEP (en service de 1989 à 2000) ne l'a pas découvert, imposant ainsi $\mathrm{m}_{\mathrm{H}}>114 \mathrm{GeV}$. La cohérence de la théorie impose par ailleurs que $\mathrm{m}_{\mathrm{H}}$ soit inférieure à environ $800 \mathrm{GeV}$.Au LHC, on recherchera donc des événements caractéristiques de la production d'un Higgs pour des masses appartenant à toute cette gamme. Le Tévatron, collisionneur protonantiproton actuellement en service à Chicago, le recherche aussi activement, mais n'a accès qu'à la gamme 114-200 GeV [2].

Pour une masse donnée, on connait tout du Higgs, en particulier ses modes de désintégration et leurs probabilités relatives ; on cherchera donc à mettre en évidence un mode de désintégration à la fois assez abondant et souffrant de peu de bruit de fond.

Ainsi, pour $\mathrm{m}_{\mathrm{H}}>2 \mathrm{~m}_{\mathrm{Z}}$ (où $\mathrm{m}_{\mathrm{Z}}=91 \mathrm{GeV}$ est la masse bien connue du boson $Z^{0}$ ), le Higgs peut se désintégrer en deux bosons W
(70\% des cas) ou deux $Z^{0}(30 \%)$. Le $Z^{0}$ a lui-même des modes de désintégration en $\mathrm{e}^{+}-\mathrm{e}^{-}$ou $\mu^{+-} \mu^{-}$(3\% chacun). Par conséquent, dans $0,1 \%$ des cas, un Higgs a pour état final 4 électrons, ou 4 muons, ou 2 électrons et 2 muons, un état final très caractéristique, très peu produit par d'autres processus, et facile à mesurer avec précision. Ce processus "plaqué or " garantirait la découverte d'un boson de Higgs standard par Atlas et CMS en un an de fonctionnement.

Les mesures actuelles des masses du boson W et du quark top permettent (par l'effet de corrections quantiques) une détermination indirecte de la masse du Higgs, qui favorise les basses masses : de la limite directe $(114 \mathrm{GeV})$ à environ $200 \mathrm{GeV}$. Dans cette gamme, le canal $\mathrm{H} \rightarrow \mathrm{ZZ}$ n'est pas dominant, et on doit s'intéresser à d'autres modes de désintégration, plus difficiles à observer, comme $\mathrm{H} \rightarrow \mathrm{WW}$ ou $\mathrm{H} \rightarrow \gamma \gamma$ (photon-photon). Les productions associées Higgs $+\mathrm{W}$, Higgs + top + antitop seront aussi recherchées. Cette détermination indirecte, valide seulement dans le cadre strict du modèle le plus simple, renforce l'intérêt porté à cet intervalle de masse, mais ne doit pas faire négliger les autres possibilités.

De très nombreuses études ont été faites à l'aide de simulations par les collaborations Atlas (fig. 3) et CMS. Leur conclusion est qu'un boson de Higgs standard ne peut échapper aux recherches après trois ans de fonctionnement du LHC.

\section{Au-delà du Modèle Standard}

Le Modèle Standard est aujourd'hui parfaitement confirmé par toutes les mesures dans le domaine des particules élémentaires. Il a pourtant d'importantes limitations (au-delà de la découverte du Higgs)

- Sa relative complexité :

pourquoi trois familles de particules ? pourquoi ces structures de symétrie ? pourquoi ces valeurs de paramètres libres (27 au total)?

- L'extrapolation à haute énergie : des problèmes théoriques difficiles apparaissent si l'on tente d'extrapoler le fonctionnement du modèle à des énergies très élevées (très loin de ce qu'on peut atteindre en laboratoire). Le modèle est donc nécessairement incomplet.

\section{Une expérience sur collisionneur : l'exemple d'Atlas}

encadré?

Les collisions ont lieu dans une petite région sur l'axe des faisceaux (quelques $\mu \mathrm{m}$ de diamètre, quelques $\mathrm{cm}$ de long). Le but de l'expérience est de reconstituer le point exact de l'interaction, la direction des particules qui en sont issues, leur impulsion, et si possible leur nature.

L'expérience est constituée d'un ensemble de détecteurs arrangés en couches concentriques autour de l'axe des faisceaux (pour la partie centrale), et de deux " bouchons » qui ferment le cylindre aux deux extrémités. Chaque détecteur est un ensemble répétitif de très nombreux détecteurs individuels, de façon à individualiser les particules. Un ensemble de champs magnétiques est fourni par des électroaimants supraconducteurs.

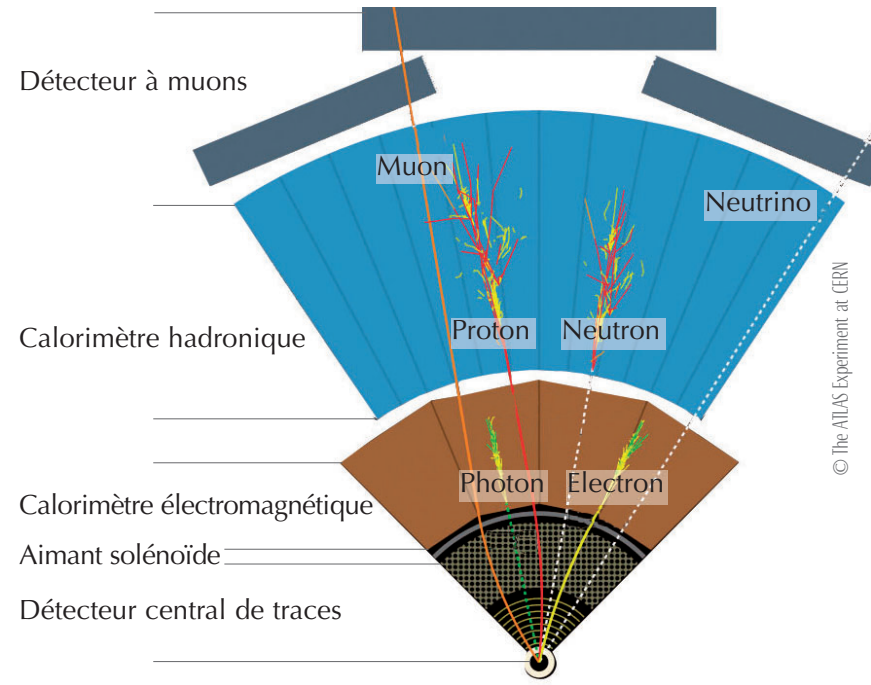

Schéma simplifié des méthodes de détection dans l'expérience Atlas, en vue transverse. Les trajectoires en pointillés ne sont pas détectées.
Certaines couches sont simplement sensibles au passage d'une particule chargée et mesurent la position de ce passage (hit). C'est le cas des couches les plus internes (sur la figure : « détecteur de traces »). Dans cette région centrale, règne un champ magnétique uniforme parallèle à l'axe des faisceaux, fourni par un solénoïde. À partir des hits, le logiciel de reconstruction peut reconstruire la trajectoire en hélice de chaque particule et en déduire son impulsion, proportionnelle au rayon de I'hélice. Parmi les particules chargées, seuls les muons atteignent la région la plus externe, où règne un champ torö̈dal fourni par le grand aimant externe à huit bobines, les autres ayant été arrêtées avant par les calorimètres. Les calorimètres sont constitués d'un assemblage de matière très dense (plomb, cuivre, fer) et de milieux détecteurs. Une particule incidente interagit avec la matière dense, engendrant des secondaires, qui elles-mêmes interagissent etc., jusqu'à ce que l'énergie des secondaires ne leur permette plus d'interagir. Le milieu détecteur interposé est sensible aux particules chargées de cette " gerbe ». La somme des signaux mesurés pour une gerbe est proportionnelle à l'énergie de la particule incidente. Noter que les calorimètres peuvent mesurer l'énergie de particules incidentes neutres. Les tailles latérale et longitudinale de la gerbe dépendent de la nature de la particule, et les détecteurs exploitent cette différence. Le calorimètre électromagnétique mesure ainsi les gerbes fines des électrons et des photons. Le calorimètre hadronique mesure les gerbes plus étendues des hadrons. Certaines particules peuvent ne pas interagir avec aucun des détecteurs, par exemple les neutrinos, ou de nouvelles particules neutres stables. Mais leur absence peut être vue dans le bilan global des impulsions transverses, puisque l'impulsion transverse de l'état initial est nulle.

Compte tenu du taux des collisions (40 millions par seconde), tous ces détecteurs doivent donner leur signal rapidement (10 à 50 ns). Le traitement et le filtrage de ces informations est en soi un défi technologique. 


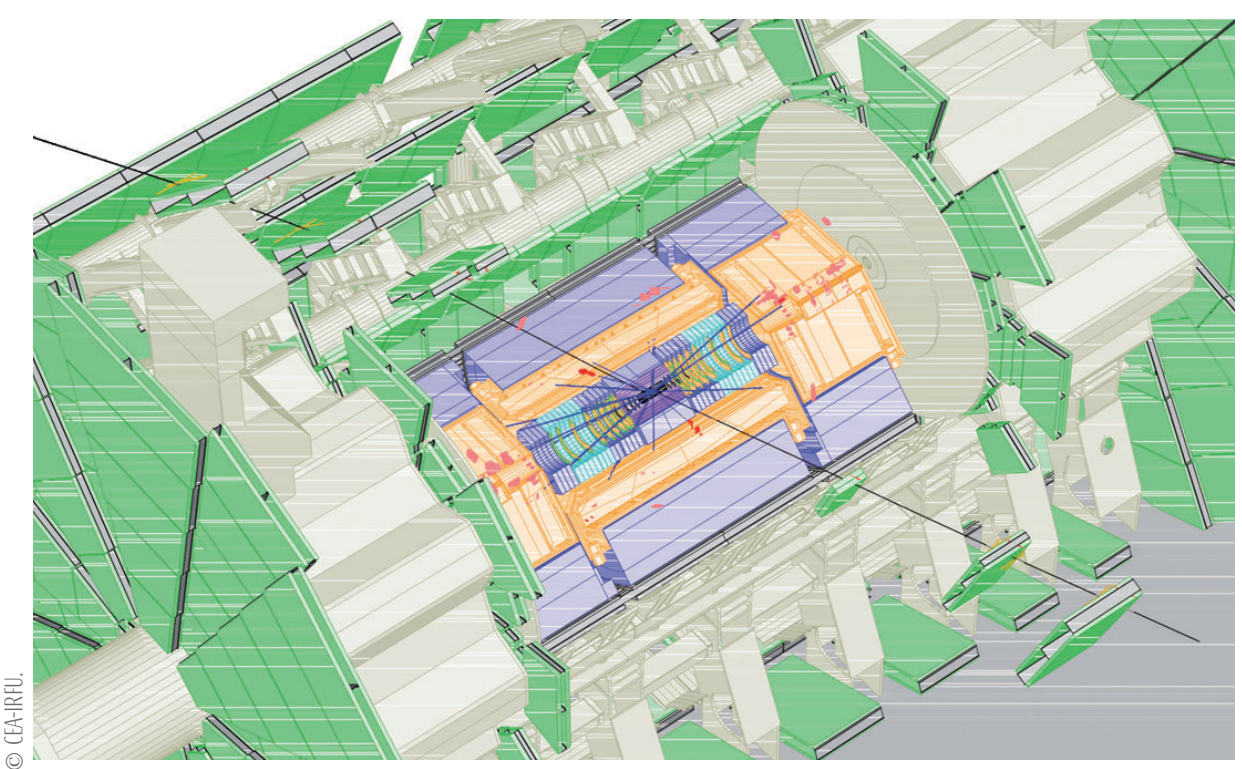

3. Simulation d'un événement Higgs $\rightarrow \overline{Z Z} \rightarrow \mathrm{e}^{+} \mathrm{e}^{-} \mu^{+} \mu^{-}$dans Atlas (écorché). On distingue les traces des deux muons qui sortent du détecteur, et les impacts des deux électrons (en rouge) dans le calorimètre central (orange).

- La matière et l'énergie noires. Depuis une dizaine d'années, l'analyse d'observations cosmologiques a conduit à réaliser que la matière ordinaire (celle du Modèle Standard) ne constitue que $4 \%$ de la densité d'énergie dans l'univers. $23 \%$ seraient une forme de matière différente, le reste (73\%) une forme d'énergie non-matérielle totalement inconnue [3].

De nombreuses théories ont été imaginées pour dépasser le Modèle Standard et tenter de répondre à ces questions. Même si découvrir un boson de Higgs standard serait un grand succès, de nombreux physiciens espèrent en fait que le LHC mettra en évidence une physique au-delà du Modèle Standard, en confirmant par exemple l'une des théories envisagées, ou même en révélant une surprise.

Pour n'en citer qu'une, la «Supersymétrie », théorie proposée dès les années 1970, mais jamais encore confirmée, apporte des réponses partielles aux questions ci-dessus. La particule supersymétrique la plus légère (LSP, pour Lightest Supersymmetric Particle) est neutre, stable, et pourrait constituer les $23 \%$ de "matière noire » de l'univers. Pour certaines valeurs des paramètres de la théorie, cette particule serait produite dans les collisions du LHC, et il serait possible de la détecter : neutre, stable, les détecteurs n'y sont pas directement sensibles, mais l'impulsion qu'elle emporte apparaîtrait comme "manquante » dans le bilan de la réaction. La concordance d'une détection au LHC avec les observations astronomiques (et pourquoi pas avec une détection au sol par une expérience de type Edelweiss [4]) serait un vrai rêve de physicien.
En tout état de cause, toutes les théories actuellement imaginées ont été explorées à l'aide de simulations, et les expériences ont été conçues pour être sensibles à leurs éventuelles manifestations.

\section{l'état des lieux du LHC}

Le 10 septembre 2008 a eu lieu la mise en service du LHC, événement très médiatisé, qui marquait l'aboutissement d'une vingtaine d'années d'efforts. Pour la première fois, un paquet de protons de $450 \mathrm{GeV}$ a été injecté dans un anneau et en a fait le tour complet, puis la même opération a été effectuée pour l'autre anneau de sens opposé. Ces premiers tours rapidement maîtrisés, et les mesures effectuées, ont démontré la qualité de la machine et l'excellente compréhension de son comportement.

Le 19 septembre 2008 est arrivé un incident majeur : suite à l'échauffement d'une jonction de câbles supraconducteurs, une grande quantité d'hélium a été vaporisée, entraînant d'importants dommages mécaniques. Après examen des enregistrements, les ingénieurs ont trouvé des signes avantcoureurs du problème initial, et pensent être à même de prévenir un autre incident de ce type. Pendant les prochains mois, il faudra bien entendu faire les réparations, améliorer le système de détection de surchauffe des supraconducteurs (en particulier les connexions), et éventuellement effectuer des modifications des dispositifs de sécurité pour en accroître l'efficacité. Ce programme ne présente pas de difficulté fondamentale, mais est assez lourd en termes logistiques. Le fonctionnement devrait reprendre à l'automne 2009.
Ce retard n'est pas en soi bien important comparé à la durée du projet, lancé officiellement en 1994. Mais la concurrence existe ! L'accélérateur de Fermilab aux USA, le Tevatron, qui fournit des énergies de faisceaux bien moins élevées $(1 \mathrm{TeV}+1 \mathrm{TeV})$ fonctionne en effet à plein régime depuis plusieurs années, et ses deux expériences (CDF et DØ) bénéficient d'une excellente connaissance de leurs détecteurs. Compte tenu de son énergie, le Tevatron pourrait détecter un premier signe (2 à 2,5 déviations standards) si le boson de Higgs a une masse comprise entre 114 et $200 \mathrm{GeV}$, précisément celle indiquée par la détermination indirecte [2]. Toutefois, une véritable découverte (5 déviations standards) ne semble pas à sa portée.

En attendant les premières collisions du LHC, les physiciens améliorent les expériences et la compréhension des détecteurs, par exemple en mesurant le passage de rayons cosmiques. Ils préparent les logiciels à l'aide de simulations et testent les transferts massifs de données, en particulier avec les "grilles de calcul ». Tous ont bien sûr hâte d'analyser les premiers événements, et d'ouvrir une nouvelle page de l'histoire de la physique de l'élémentaire.

\section{Pour en savoir plus...}

\author{
1 - M. Jacob, « Une brève histoire du CERN », \\ Bulletin de la SFP 147 (2005) 17-20. \\ 2 - Voir l’article de G. Bernardi dans ce numéro, \\ pp. 10-14. \\ $3 \bullet$ V. Ruhlmann-Kleider, Reflets de la physique $\mathbf{1 2}$ \\ (2009) 16-19. \\ 4 - Voir http://edelweiss2.in2p3.fr/ \\ Bulletin de la SFP 155 (2006) 15-20. \\ www.cern.ch ,et en particulier \\ http://public.web.cern.ch/public/en/LHC/LHC-en.html \\ En français, le site $\boldsymbol{h t t p : / / w w w . / h c - f r a n c e . f r / ~}$ \\ présenté par les équipes françaises.
}

\section{Revues:}

- " De quoi est fait l'Univers ? », Dossier Pour La Science, 62 (janvier-mars 2009), en particulier pp. 8 à 27.

- La Recherche, 424 (novembre 2008), pp. 31 à 38 (3 articles).

- « LHC : la naissance d’un géant », Science \& Vie, Hors-Série nº 244 (septembre 2008).

- « La nouvelle conquête de l'infiniment petit », série d’articles, Sciences et Avenir, 735 (mai 2008).

- "Des géants pour traquer l'infiniment petit", Pour La Science, 361 (novembre 2007) 98-104.

Livre:

LHC : enquête sur le boson de Higgs, de Michel Davier, aux éditions du Pommier (2008). 